\title{
TASKRADAR: TASK VISUALISATION AND MONITORING WITHIN AUTOMOTIVE PRODUCT DEVELOPMENT LIFECYCLE USING SEMANTIC TECHNOLOGIES
}

\author{
Selver Softic ${ }^{1}$, Manfred Rosenberger ${ }^{2}$, Ioan Turcin', Alexander Stocker ${ }^{2}$
}

1 Campus 02 University of Applied Sciences, Körblergasse 126, 8010 Graz, Austria, e-mail: selver.softic@ campus02.at, ioan.turcin@campus02.at

2 Virtual Vehicle Research Center, Inffeldgasse 21A, 8010 Graz, Austria, e-mail: manfred.rosenberger@v2c2.at, alexander.stocker@v2c2.at

Received: 2016.06 .04 Accepted: 2016.07.05 Published: 2016.09.01

\begin{abstract}
Project management within the automotive production in specific departments is still done separately and does not interact with engineering process. Our work aims on providing flexible data insights on collaboration tasks within such environments. We apply semantic technologies RDF, OWL and SPARQL with a specific domain related ontology PROTARES (PROject TAsks RESources) to interlink, describe and query domain knowledge. As proof of concept we are introducing an experimental visualisation interface called TaskRadar. Our application resides on domain ontology and allows knowledge based browsing and visualisation of tasks in development process. With this example we want to show, how semantically driven customized views can support monitoring and reflection as well as decision-making within the early phases of the automotive product lifecycle.
\end{abstract}

Keywords: product lifecycle management, semantic web, munfacturing, monitoring, information visualisation

\section{INTRODUCTION}

The timescale for development of new cars has been drastically shortened, the several vehicle variants has multiplied over time. Car manufacturers are doing their business globally with a large number of suppliers and retailers. Product development relies on cross-company collaboration and implies intensive communication of product data [15]. Such exchange, however, is still carried in face to face meetings.

Product Lifecycle Management (PLM) faces a challenge: how to serve involved stakeholders with adequate information to support their decisions and decrease at the same time the administrative effort. A PLM system aims at delivering views on time, stakeholders, components and processes at once.
The idea that inspired our approach is that overall understanding of the product system, leads to improvement in decision-making [3] as well to more formal access to the knowledge within the development process [16]. New technologies like Semantic Web could fill the missing technology gap to enable context based modeling as prerequisite for focused and more eficient communication on product development.

Our approach uses semantic technologies to structure and describe the collaboration on the task level. SPARQL [21] queries deliver preprocessed data for visualisation, which creates selective views on product development tasks including links to involved engineering objects. In this way a visualisation contributes better task analysis and monitoring within development process. 


\section{RELATED WORK}

The Product Lifecycle Management (PLM) aims at improving and monitoring development processes in automotive product lifecycle. It assumes that adequate tools, standards and technologies when involved into the product lifecycle can help the manufacturers to increase their product quality and competitiveness as well as to improve the maintenance. This approach leads to remarkable improvements $[5,15]$.

A PLM system requires a high level of coordination and integration as stated in [11]. As the key enabler for PLM solutions have been identified entities like: stakeholders, tools, actions, and relations between them [4]. Formalising these relations contributes data reuse as well as context based consumption of information [9].

Application of ontologies in production management delivers more flexibility than formerly used approaches [10, 12, 13]. The potential of Semantic Web and its technology stack [2] to rise the tacit knowledge is used to optimise particular segments of product lifecycle $[17,19]$. The efforts in this field so far were focused to model partial aspects of product lifecycle like maintenance [12] or product description [13]. However, to explore serendipity and contribute lifecycle improvement, aspects of organisation, communication and interaction which include entities, stakeholders, events, process states and relations among them have to be considered as well.

Semantic technologies are able to design such complex relations between the entities, and they respond flexible on changes in context of information as well. Further they incorporate huge inference potentials $[18,1]$, what makes them an approved choice for current effort.

\section{METHODOLOGY}

In following we want to introduce the essential use case for semantically driven "Task Visualisation and Monitoring" within automotive product lifecycle. This use case involves resources, repositories, targets, persons and time spent on certain task as well as requirements related to them. As experimental data-

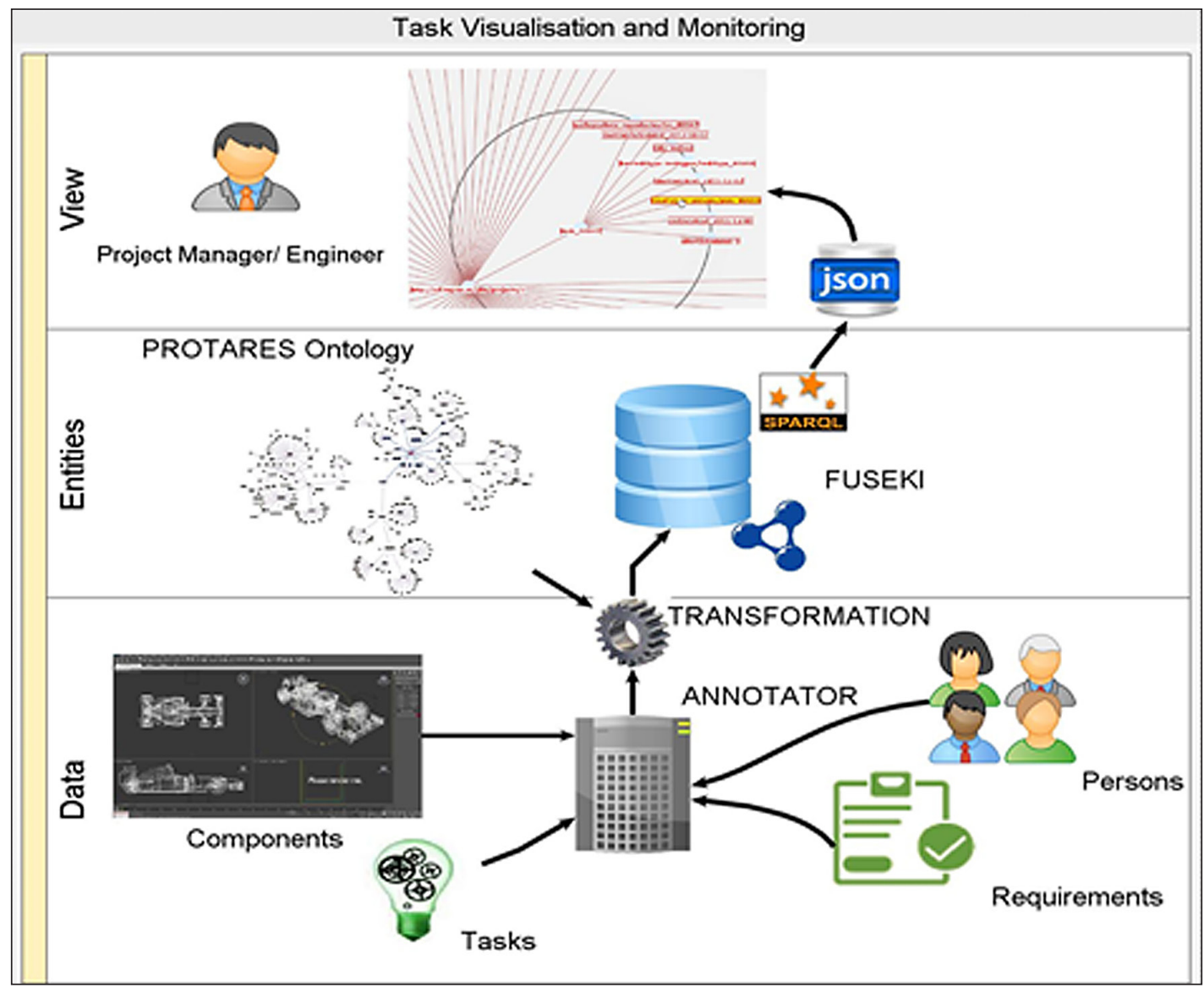

Fig. 1. Implementation architecture (11 point) 


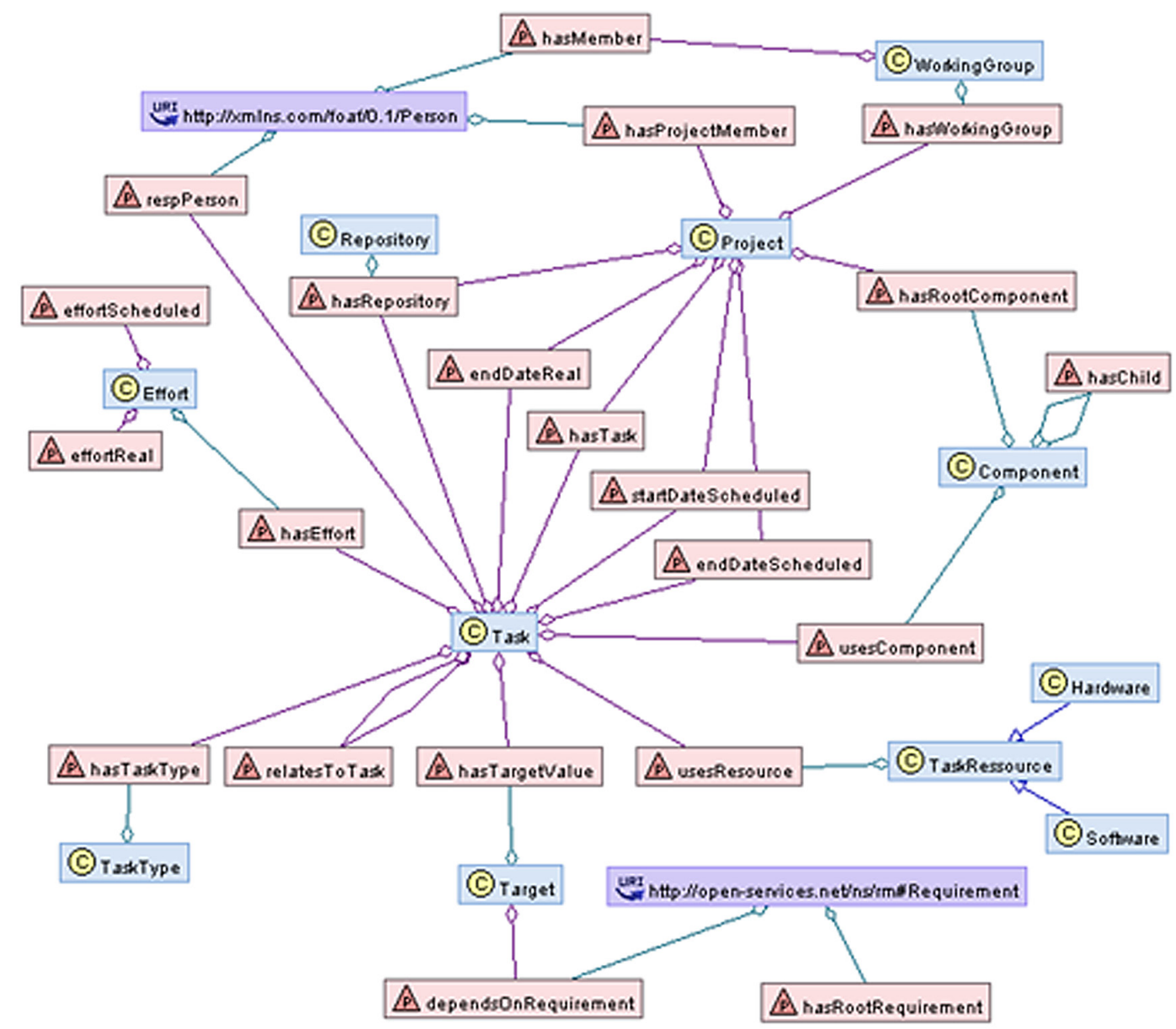

Fig. 2. PROTARES - Ontology for activity modelling with all classes and properties

set we use data from our local formula student university racing team.

Every year "TU Graz Racing Team" takes part in the FSAE Formula Student [23] competition with a self developed and produced racing car. We used their data, experience and knowledge to define together the "Task Visualisation and Monitoring" use case. The reference data set has information about around 700 requirements, 60 tasks, 358 related components, 24 resources and 21 persons involved into development process.

\section{IMPLEMENTATION}

Enquiry on existent works [14] about ontologies and formal approaches $[6,12,13,16]$, delivered incomplete results and offered none model with suficient number of properties. Lifecycle [24] and TOVE Ontology [25] project [7, 8] are covering only partially our needs. They are upper level ontologies with insuficient granular precision required to describe resources in our use case.
In order to enable very precise modelling we decided to create a new domain ontology named PROTARES (PROject TAsks RESources) presented with object related properties in Figure 2. The PROTARES [22] combines own concepts with wide used FOAF (Friend of A Friend) [26] ontology and Requirements Management Ontology from OSLC (Open Services for Lifecycle Collaboration) initiative [27].

\section{PRELIMINARY RESULTS}

The most important process which consider data model occurs in transformation module of annotator application (Figure 1). The structured data in annotator application is mapped from native XML structure to the PROTARES domain ontology (Figure 2). The data within is transformed into RDF instances as depicted in Figure 3. Tables and graphs should be legible and in reproducible quality (including in text) with references in text.

Storing data about engineering artifacts in RDF [20] enables revealing different views on 


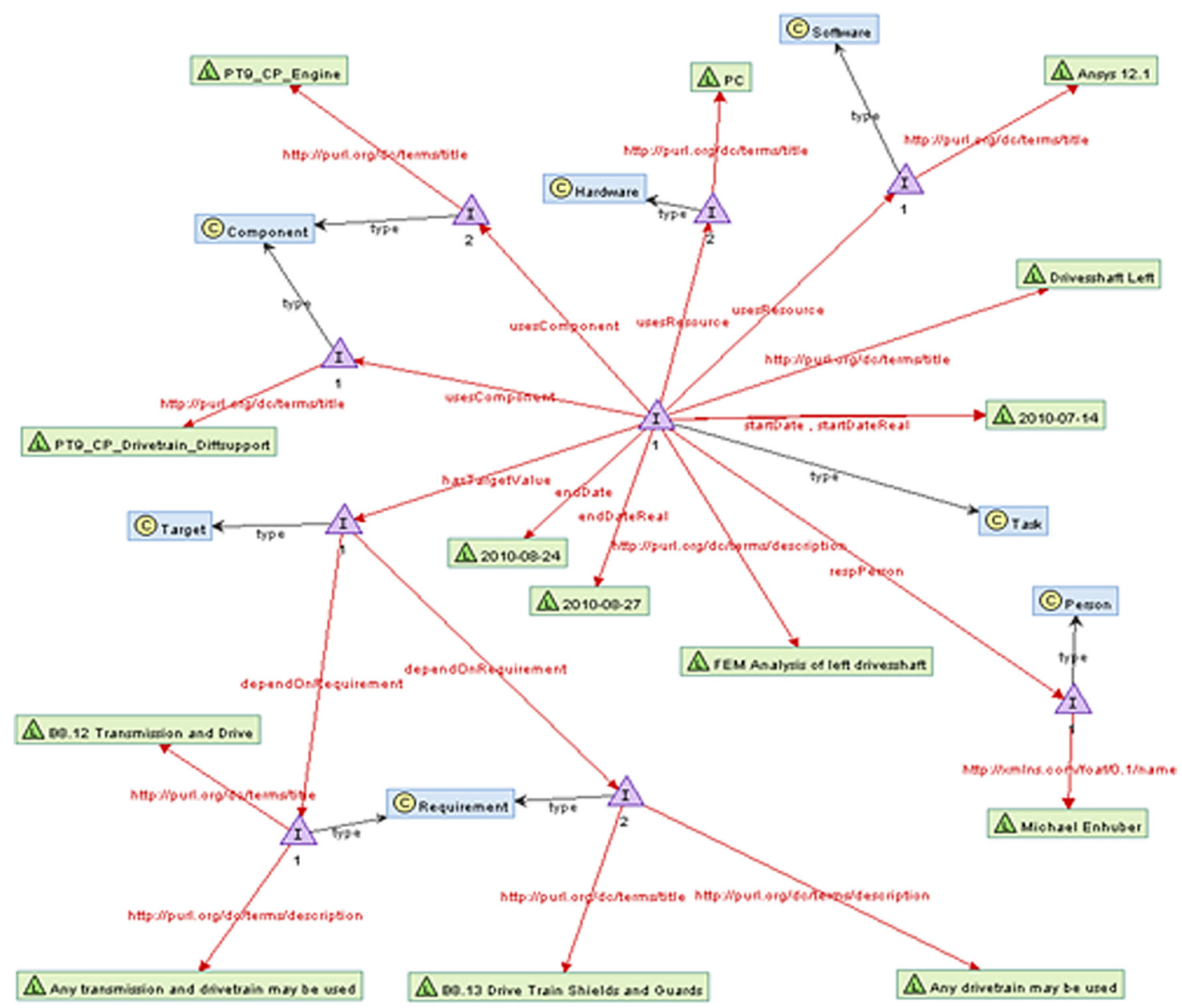

Fig. 3. Sample instance of PROTARES Ontology

data relations and makes this data easier and more flexible retrievable. The triple store we used supports the generation of results in various standard formats like XML, CSV or JSON. We use JSON results of queries within our application called "TaskRadar" residing on the top of architecture stack which visualises the task related information for the end-user.

Figure 4 demonstrates with a sample query how easily the data from RDF graphs can be retrieved. In this example the query delivers all components from all tasks with cetrain word in the title.

Figure 5 shows the preliminary browsing interface called "TaskRadar". The application visualises tasks from automotive development project as expandable nodes within radial interface. Line between the nodes represent direct relation. Focused node is always in the center of interface and its branches are outlined to reveal

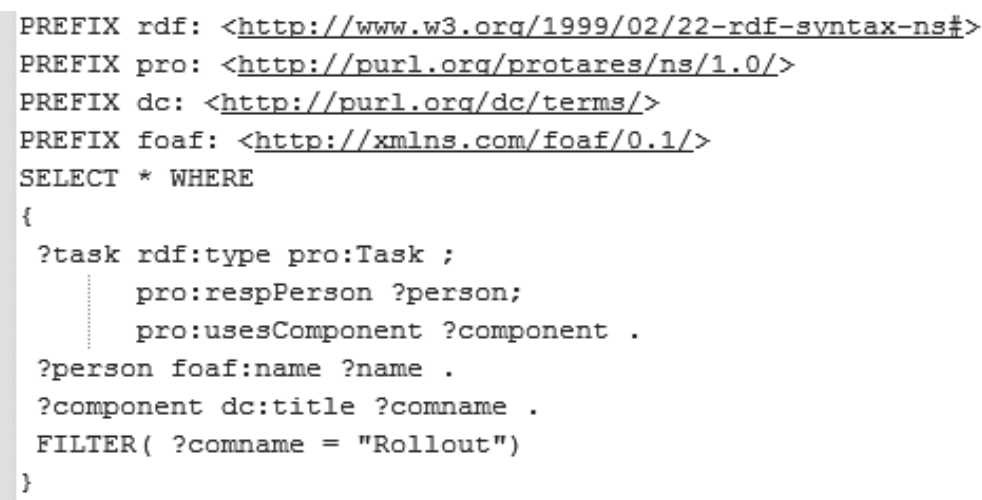

Fig. 4. Retrieving all information about specific task 


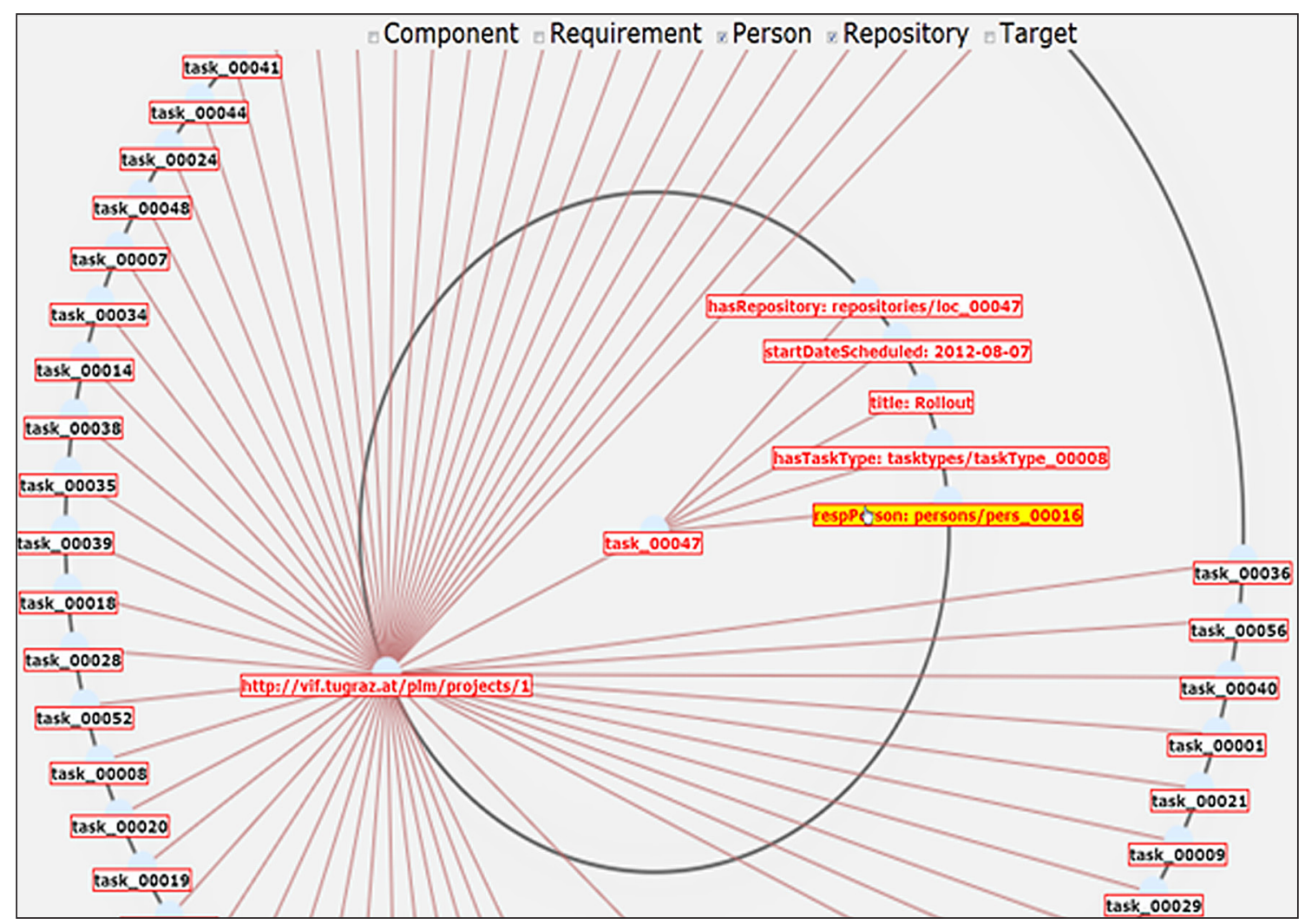

Fig. 5. "TaskRadar" graphical user interface showing single task information in focus

the information context. Circles around the center represent the depth of relation. With "TaskRadar" a user is able to browse the tasks as well to filter the view on tasks and their relations. Dependently on which filters are active the view delivers either partial all holistic view on tasks and project. All inputs for the "TaskRadar" are created with SPARQL queries like the one in Figure 4. Interface is JavaScript based and uses JSON [28] formatted results from SPARQL Endpoint running on a FUSEKI [29] server.

\section{CONCLUSIONS}

Ontology based interlinking of engineering objects with participants and resources allows getting an overview on product development. The software prototype "TaskRadar" gives an idea how this queries can be visualised on benefit of participants in the development process.

Documents and other data objects like product components or requirements are managed in Document Management Systems (DMS), Product Data Management (PDM) or Requirement Management Systems (RQM). Information of these systems is still required to display all the relations of a task. Interfaces to the named systems can help to use this data. Such improvement requires a review of PROTARES ontology.

Further, a survey which includes questions about aspects such as content, navigation and in-formation model will be conducted to measure and capture overall usability. By implementing the first prototype of "TaskRadar" as proof of concept for main goal of browsing and monitor-ing of tasks in an automotive product development lifecycle, we showed that Semantic Web with suitable choice of problem domain description, delivers satisfying, scalable results and acts as enabler for context based visualisations.

\section{Acknowledgements}

The authors would like to acknowledge the financial support of the "COMET K2 - Competence Centres for Excellent Technologies Programme" of the Austrian Federal Ministry for Transport, Innovation and Technology (BMVIT), the Austrian Federal Ministry of Economy, Family and Youth (BMWFJ), the Austrian Research Promotion Agency (FFG), the Province of Styria and 
the Styrian Business Promotion Agency (SFG). We would furthermore like to express our thanks to our supporting industrial and scientific project partners, namely "TUG Racing Team" at the Graz University of Technology.

\section{REFERENCES}

1. An Y., Borgida A., Mylopoulos J.: Inferring complex semantic mappings between relational tables and ontologies from simple correspondences. In: OTM Conferences (2), vol. 3761 of Lecture Notes in Computer Science, 2005, 1152-1169.

2. Berners-Lee T., Hendler J., Lassila O.: The Semantic Web: Scientific American. Scientific American, May 2001.

3. Bruun H.P.L., Mortensen N.H.: Visual product architecture modelling for structuring data in a plm system, In: PLM, vol. 388 of IFIP Advances in Information and Communication Technology, 2012, 598-611.

4. Denger A., Unzeitig W.: Future product lifecycle management (plm) - a con-sideration of informal communication as a key enabler for future product develop-ment, In: PLM, vol. 388 of IFIP Advances in Information and Communication Technology, 2012, 435-444.

5. Dubois M., Hamel L., Gardoni M.: Creative tools and processes to remain competitive in the twentyrst century, In: PLM, vol. 388 of IFIP Advances in Information and Communication Technology, pp. 332-338. Springer, 2012.

6. Fortineau V., Paviot T., Louis-Sidney L., and Lamouri S. Swrl as a rule language for ontolo-gybased models in power plant design, In: PLM, vol. 388 of IFIP Advances in Information and Communication Technology, 2012, 588-597.

7. Fox M.S.: The tove project towards a commonsense model of the enterprise, In: Proceed-ings of the 5th international conference on Industrial and engineering applications of artifi-cial intelligence and expert systems, IEA/AIE '92, London, UK, 1992, 25-34.

8. Fox M.S., Barbuceanu M., Gruninger M..: An organisation ontology for enterprise modeling: Preliminary concepts for linking structure and behaviour, Computers in Industry, 29(1-2), 1996, 123-134.

9. Hani M.A.E., Rivest L., Maranzana R.: Product data reuse in product development: A practi-tioner's perspective, In: PLM, vol. 388 of IFIP Advances in Information and Communica-tion Technology, 2012, 243-256.
10. Jun H., Kiritsis D., Xirouchakis P.C.: A primitive ontology model for product lifecycle meta data in the closed-loop plm. IESA, 2007, 729-740.

11. Jun H.-B., Shin J.-H., Kiritsis D., Xirouchakis P.C.: System architecture for closed-loop plm, Int. J. Computer Integrated Manufacturing, 20(7), 2007, 684-698

12. Matsokis A., Hedi Karray M., Chebel-Morello B., Kiritsis D.: An Ontology-based model for providing Semantic Maintenance, In: 1st IFAC Workshop on Advanced Maintenance Engi-neering, Services and Technology. Lisbonne, Portugal, 2010, 17-22.

13. Matsokis A., Kiritsis D.: An ontology-based approach for product lifecycle management. Computers in Industry, 61(8), 2010, 787-797.

14. Mostefai S., Bouras A.: What ontologies for plm: a critical analysis, In: 12th International Conference on Concurrent Enterprising, K.-D. Thoben, K.S. Pawar, Taisch, \& S. Terzi, (Eds.), 26-28 June 2006, Milan, Italy.

15. Müller P., Pasch F., Drewinski R., Bedenbender H., Hayka H., Stark R.: Study on collabora-tive product development and digital engineering tools, In: PLM, 2012, 389-399.

16. Patil L., Dutta D., Nistir R.D.S., Sriram, R.D., Gutierrez C.M.: Ontology formalization of product semantics for product lifecycle management, 2005.

17. Rivest L., Bouras A., Louhichi B.: Product Lifecycle Management. Towards Knowledge-Rich Enterprises, vol. 388 of IFIP Advances in Information and Communication Technolo-gy. Springer, 2012.

18. Wache H., Vögele T., Visser U., Stuckenschmidt H., Schuster G., Neumann H., Hübner S.: Ontology-based integration of information - a survey of existing approaches, In: IJCAI'01 Workshop: Ontologies and Information Sharing, 2001, 108-117.

19. Young R., Gunendran G., Cutting-Decelle A., Gruninger M.: Manufacturing knowledge sharing in plm: A progression towards the use of heavyweight ontologies, International Journal of Production Research, 45(7), 2007, 1505-1519.

20. http://www.w3.org/RDF/

21. http://www.w3.org/TR/rdf-sparql-query/

22. http://purl.org/protares/ns/1.0/

23. http://students.sae.org/competitions/formulaseries/

24. http://vocab.org/lifecycle/schema

25. http://www.eil.utoronto.ca/enterprise-modelling/ tove

26. http://xmlns.com/foaf/spec/

27. http://open-services.net/ns/rm

28. http://www.json.org/

29. http://jena.apache.org/ 